\title{
TB or not TB: an unusual sore finger
}

\author{
D Sunderamoorthy, V Gupta, A Bleetman
}

\begin{abstract}
A 25 year old Afro-Carribean presented to the accident and emergency department with a painful swelling of the little finger. Radiology revealed periosteal elevation and a bone scan showed a hot spot in the proximal phalanx, suggestive of a tumour or an infection. He gave a history of recurrent chest infections in the past, the chest radiograph revealed apical lesions and loculated pleural effusion. Bronchoscopy washings were positive for acid fast bacillus suggestive of pulmonary tuberculosis with tuberculous dactylitis. A detailed history and high index of suspicion is important as delay in diagnosis can have serious consequences for the patient. (Emerg Med f 2001;18:490-491)
\end{abstract}

Keywords: tuberculosis; tuberculous dactylitis

Tuberculous dactylitis is a rare musculoskeletal infection. The diagnosis is often delayed becuase of unfamiliarity with the condition. ${ }^{1}$ We report a case of tuberculous dactylitis in a 25 year old Afro-Carribean man. The clinical presentation, diagnosis and the differential diagnosis are discussed.

\section{Case report}

A 25 year old Afro-Carribean man presented to the accident and emergency (A\&E) department with a history of painful swelling of the right little finger of two months duration. $\mathrm{He}$ denied a history of trauma. Examination revealed swelling and tenderness of the proximal phalanx and the metacarpophalangeal joint of that finger. There was restricted movement at the metacarpophalangeal joint.

Radiographs of the right hand showed soft tissue swelling, periosteal elevation and osteopenia of the proximal phalanx of the little finger (fig 1). A differential diagnosis of tumour or of infection was considered and an orthopaedic opinion was sought. Bone scan was recommended. This demonstrated a hot spot in the right little finger (fig 2). Blood tests showed an increase erythrocyte sedimentation rate $(36 \mathrm{~mm} 1 \mathrm{st} \mathrm{h})$ and $C$ reactive protein (55 $\mathrm{mg} / \mathrm{l})$ with a normal white cell count $(6.3 \times$ 1000/1).

The patient was reviewed in the A\&E review clinic with these results after two weeks. By this time he had developed a temperature of $37.7^{\circ} \mathrm{C}$. A more detailed history showed that he had been experiencing a dry cough for the past six months. He also reported intermittent chest pain. There was a past history of chest infections that had been treated with antibiotics. A chest radiograph demonstrated patchy

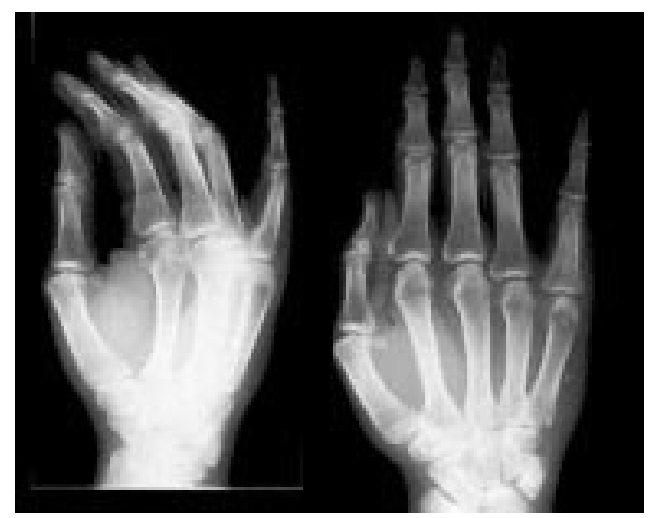

Figure 1 Radiograph of the right hand showing periosteal elevation and osteopaenia of the proximal phalanx of the little finger.

RT

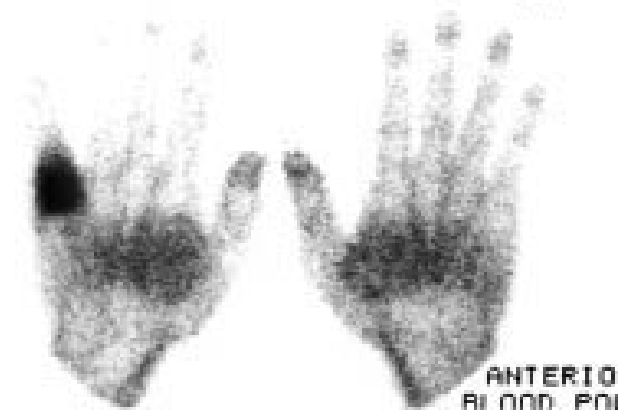

Figure 2 Bone scan showing hot spot in the proximal phalanx of the little finger.

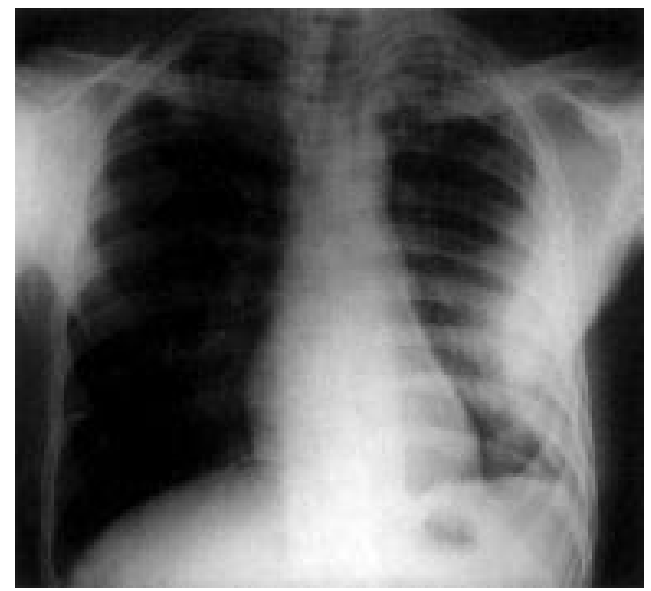

Figure 3 Radiograph of the chest showing apical shadows in the lung with loculated effusion in the left lung.

shadowing in both the apices with a loculated left sided effusion (fig 3 ).

A diagnosis of pulmonary tuberculosis with tuberculous dactylitis was now suspected. The patient was transferred to the infectious disease unit for further management. Bronchoscopy washings were positive for acid fast bacillus and cultures revealed mycobacterium
Accepted for publication 15 March 2001 
tuberculosis, which proved sensitive to isoniazid, rifampicin and ethambutol. Antituberculous medication was prescribed and the patient was discharged to out patient follow up.

\section{Discussion}

In industrialised countries one third of the new cases of extra pulmonary tuberculosis occur in the immigrant population. ${ }^{23}$ Tuberculous dactylitis accounts for $4 \%$ to $8 \%$ of skeletal tuberculosis. ${ }^{4}$ It is relatively common in children aged between 1 and 6 years and in adults between the ages of 20 and $50 .{ }^{13}$ It is rare in children over 10 years of age. ${ }^{1}$ It is three times more common in men than in women. ${ }^{3}$

\section{CLINICAL PRESENTATION}

The usual presenting feature is painless swelling of a finger. ${ }^{15} \mathrm{~A}$ single digit, usually middle or index finger, is involved. The proximal phalanx is most often involved. ${ }^{1}$ Involvement of the toes is less common. Multiple sites are involved in $25 \%$ to $30 \%$ of cases. ${ }^{3}$ Joint involvement may or may not be present. Fistulas and sinuses occur in neglected cases.

\section{RADIOLOGICAL FEATURES}

The diagnosis should always be considered in cases of unexplained swelling of a finger or toe-particularly in those at a higher risk of having tuberculosis. The radiological changes may be grouped in four broad categories:

- Soft tissue swelling, which is often marked and fusiform with no apparent change in neighbouring bones or joints.

- The earliest evidence of bony involvement is periosteitis indicated by linear deposition of new bone with increasing cortical thickness and involucrum formation. Gradual destruction with sequestration of the bone then occurs while the involucrum thickens. If much of the involucrum's internal aspect is absorbed a cyst like cavity remains, which appears to ballooned out or injected with air, the so called spina-ventousa.

- Diffuse uniform infiltration's with "honeycombing"-The affected phalanx may show minimal or moderate expansion and appear infiltrated throughout with honeycombed or lace-like appearance.

- Localised destruction with reactive osteitis-the phalanx shows small areas of cortical or cancellous destruction with no apparent change in the other parts of the bone. ${ }^{6}$ Diaphyseal sequestration and pathological fractures can also occur. ${ }^{17}$

\section{DIFFERENTIAL DIAGNOSIS}

The differential diagnosis includes congenital syphilis, enchondroma, pyogenic osteomyelitis, sarcoma, sarcoidosis, Paget's disease and other granulomatous infections. Tuberculous dactylitis tends to present in an older age group, whereas congenital syphilis is apparent in the first years of life, bilaterally symmetrical and not associated with soft tissue swelling or sequestration. In sarcoidosis well demarcated cystic lesions are found in the phalanges of the fingers, although bony expansion and periosteal new bone formation are not found. In enchondromatosis numerous lesions are present that are larger than the small cysts seen in tuberculosis. ${ }^{236}$

The diagnosis is confirmed by biopsy, which demonstrates a granulomatous reaction with central caseous necrosis and Langhans type giant cells. Acid fast bacilli are demonstrable by the Ziel Neilsen technique and cultures will reveal the sensitivity of the infecting organism.

The treatment is specific antimicrobial therapy with surgical excision if necessary. ${ }^{37}$

Tuberculosis is being diagnosed more frequently in the developed world ${ }^{2}$ and should be considered in a patient presenting with a swollen finger of insidious onset. This case demonstrates the importance of a detailed history in arriving at the diagnosis. Entertaining a high index of suspicion is important as delay in diagnosis can have serious consequences for the patient.

We gratefully acknowledge the help of Dr Aidan MacNamara, consultant in Accident and Emergency, Heartlands Hospital, in preparing this case report.

Mr A Bleetman is guarantor of this paper.

Funding: none.

Conflicts of interest: none.

1 Abdelwahab IF, Lewis MM, Hermann G. Case Report 528 : Tuberculous dactylitis (right great toe). Skeletal Radiol Tuberculous dacty

2 Clarke JA. Tuberculous dactylitis in childhood. The need for continued vigilance. Clin Radiol 1990;42:287-8.

3 Jensen CM, Jensen CH, Paerregaard A. A diagnostic problem in tuberculous dactylitis. $\mathcal{F}$ Hand Surg $[B r] ~ 1991 ; 16$ : 202-3.

4 Brazille P, Timsit MA, Quillard A, et al. Tuberculous dactylitis of a single phalanx. [Letter]. Rev Rhum Engl Ed 1998;65:511-12.

5 Leung PC. Tuberculosis of the hand. Hand 1978;10:28591.

6 Feldmann F, Auerbach R, Johnston A. Tuberculous dactylitis in the adult. American fournal of Roentgenology, Radium Therapy and Nuclear Medicine 1971;112:460-79.

7 Urovitz EP. Tuberculous dactylitis: a rare entity. Can f Surg 1982;25:689-90. 